\title{
A conserved arbuscular mycorrhizal fungal core-species community colonizes potato roots in the Andes
}

\author{
Carolina Senés-Guerrero $^{1}$ • Arthur Schüßler ${ }^{1}$
}

Received: 27 October 2014 / Accepted: 16 March 2015 / Published online: 31 March 2015

(C) The Author(s) 2015. This article is published with open access at Springerlink.com

\begin{abstract}
Plant-symbiotic arbuscular mycorrhizal fungi (AMF) are of high global ecological and economic importance, but describing environmental communities of AMF at the species level remains a challenge, despite the need to understand AMF-plant preferences and to apply AMF in sustainable agriculture. Here, the potato-associated AMF species community composition was assessed for three Andean countries along an altitudinal gradient and at different plant stages, by using 454 GS-FLX+ sequencing of a 760 bp LSU rRNA gene PCR amplicon. Two methods were compared: defining OTUs based on a simple sequence similarity threshold, or affiliating reference sequences to species based on a high throughput phylogenetic annotation approach using an evolutionary placement algorithm (EPA). The EPA-based approach was not only more precise, but also fundamental to robustly unveil the AMF species community composition. The principal advantage of this approach was also demonstrated by using artificially constructed datasets based on validated public database sequences. The affiliation of sequence reads to species using phylogenetic annotation revealed a surprisingly conserved AMF core-species community structure in Andean potatoes, regardless of different plant stages and environmental factors. In total, 41 species were detected and in some cases
\end{abstract}

Electronic supplementary material The online version of this article (doi:10.1007/s13225-015-0328-7) contains supplementary material, which is available to authorized users.

Carolina Senés-Guerrero

carolina.senes@gmail.com

1 Department of Biology, Genetics, Ludwig-Maximilians University, Grosshaderner Str. 4, 82152 Planegg, Martinsried, Germany more than 25 species were found colonizing an individual root system. Acaulospora species were identified as dominant colonizers, co-occurring with Cetraspora nodosa and certain Claroideoglomus and Rhizophagus species in most potato root samples.

Keywords 454 pyrosequencing · Andean ecosystems · Arbuscular mycorrhizal fungi species communities · DNA based species identification - Evolutionary placement algorithm · Solanum tuberosum (potato)

\section{Introduction}

In a world confronted with an increasing human population, one of the main challenges is sustainable food production without negative impacts on valuable natural resources. While intensive agriculture promoted during the green revolution secured food demand over the last decades, it was also accompanied by high environmental costs that are nowadays recognized as public health and food production threats (Tilman et al. 2002). As an alternative, a more sustainable agriculture respecting the positive impact of many soil microorganisms, including their potential utilization, has received significant attention (van Loon 2007; van der Heijden et al. 2008). One of the most relevant groups of such soil microbes are arbuscular mycorrhizal fungi (AMF), obligate symbionts of the vast majority of land plants, including the ten most important human food crops (Smith and Read 2008; Brundrett 2009; FAO 2012). In exchange for photosynthesis derived carbohydrates, AMF transport inorganic nutrients and water from the soil to the plants and can strongly increase the utilization efficiency of fertilizers, especially the nonrenewable phosphorus (P) (Tawaraya et al. 2012). Therefore the use of 
AMF as inoculum for agricultural purposes is promising and may be most important for P-demanding crops such as potato (Solanum tuberosum) (Dechassa et al. 2003).

Potato has a worldwide increasing value (Birch et al. 2012) and is the 4th largest food crop with a production of 365 million tons (Mt) reported in 2012, following maize, rice and wheat (FAO 2012). Cultivated potato originated in the Andean region (Spooner et al. 2005) where nowadays it is a staple crop, and therefore increasing the yield of native potato cultivars by using AMF is a topic of interest (Davies et al. 2005). However, although AMF have been found colonizing plants at high altitudes in the Andes, at the Bolivian Altiplano (Urcelay et al. 2011) and up to 5,250 m (Schmidt et al. 2008), so far there are no studies analyzing the AMF community composition of potato in Andean ecosystems. Moreover, the general knowledge about AMF species colonizing potato roots is scarce, even though a better understanding of preferential AMF-host associations would facilitate the specific selection of AMF to be used as inoculum in sustainable agricultural practices. The identification of AMF in plant roots can only be obtained by using molecular markers. Although DNA-based detection is frequently used for field samples, the taxonomic level of resolution usually is undefined and concise information of the AMF species community composition remains unknown. For molecular ecological studies, the small subunit (SSU) (Öpik et al. 2013) and/or internal transcribed spacer (ITS) (Redecker 2000) and/or the large subunit (LSU) rDNA regions (Mummey and Rillig 2007) were frequently used. Yet, due to the low variability in the SSU, an extremely high intraspecific variability in the ITS or the use of relatively short LSU fragments, most analyses using these markers led to phylogenetic resolution at an undefined taxonomic level in-between species and genus (Stockinger et al. 2009).

At present 454 GS-FLX+ amplicon-sequencing provides read lengths of up to approx. $1 \mathrm{~kb}$ and is the high throughput sequencing method providing the best phylogenetic resolution power to monitor AMF in the field. We therefore developed a 454-sequencing based process to monitor AMF species and applied it to analyze potato-associated AMF communities. The SSU-ITS-LSU rRNA gene region used was defined as an extended DNA barcode resolving closely related AMF species (Stockinger et al. 2010; Schoch et al. 2012). Importantly, it can be amplified from field samples using AMF specific PCR primers (Krüger et al. 2009), which recently were confirmed to have the broadest taxonomic coverage among other PCR primers frequently applied for AMF detection (Kohout et al. 2014). The primers amplify approx. $1500 \mathrm{bp}$ sequences, which serve to compute a robust reference sequences phylogenetic tree functioning as a "phylogenetic backbone" (Krüger et al. 2012) for the placement of the shorter, approx. $760 \mathrm{bp}$ long 454 sequences by a maximum-likelihood evolutionary placement algorithm (EPA).
The principal problem in such an approach is that a large number of unknown AMF species must be expected in uncharacterized, putatively highly diverse ecosystems. The lack of sequence information for those AMF would result in deep sequencing data impossible to be robustly affiliated to species. Therefore, we recently analyzed the potato associated AMF in the Peruvian Andes by a clone library and Sanger sequencing based approach, characterizing the above noted 1500 bp extended DNA barcode (Senés-Guerrero et al. 2014). It turned out that approximately half of the AMF species in this ecosystem were unknown. Here, the 1500 bp sequences of these formerly uncharacterized AMF now allow the computation of a phylogenetic backbone for deep sequencing analyses.

For the first time the AMF species community of a crop plant from Andean ecosystems is analyzed in depth. The main goal was to describe, at species level, the composition of the AMF community colonizing potato roots, and to determine putative main players involved in potato AM in the ecosystems studied. We wanted to i) validate our new approach by studying whether the interpretation of the AMF community structure is principally influenced by the methods used, ii) determine how many and which AMF species live in an individual root system and which AMF co-occur, and iii) analyze whether the AMF species community composition is influenced by altitude and/or plant developmental stage.

\section{Materials and methods}

\section{Sample collection}

In each of the three studied countries, Bolivia, Ecuador and Peru, potato roots were sampled at three plant developmental stages (emergence, flowering and senescence; according to the potato development system described by Hack et al. 1993), from four potato fields which were located at four different altitude ranges (from 2,658 to 4,075 $\mathrm{m}$ above mean sea level (mamsl)). Three replicate samples were analyzed but 5 replicate samples were collected at each sampling in case some replicates were PCR-negative. The whole root system corresponding to different plants was harvested at different sampling dates depending on the planting times of each country and according to the plant developmental stages, selected based on the BBCH scale of the potato development system. The five replicates from the senescent stage from one Ecuadorian field could not be sampled due to early harvesting by the field owner without notice. In total, 105 samples were analyzed by 454 sequencing ( 3 replicates per location per stage). Six different potato varieties were grown at the different locations (Bolivia: Waycha; Ecuador: Superchola, Guata, Fripapa; Peru: Yungay, Unica; see Online Resource 1 for site descriptions), but because it was not possible to sample 3 
replicates of each potato variety this is not a parameter included in our statistical analyses. Directly after sampling, the individual root systems were washed with water and cut into $1 \mathrm{~cm}$ pieces. Representative samples for each root system were placed in $80 \%$ ethanol in $10 \mathrm{ml}$ cryovials. The root material processed at the field sites and samples were later stored at $-20^{\circ} \mathrm{C}$ until DNA extraction.

\section{4-pyrosequencing}

DNA was extracted from the root samples using the FastDNA Spin Kit for soil following the manufacturer's instructions but using Lysing Matrix A tubes with an extra big ceramic bead as described in Senés-Guerrero et al. (2014). The first PCR was performed as described by Krüger et al. (2009) with the AMF specific primers SSUmAf-LSUmAr using 35 cycles and $0.5 \mu \mathrm{l}$ of the total DNA as template. The primers target a $1.8 \mathrm{~kb}$ region covering part of the SSU rRNA gene, the complete ITS region (including the 5.8S rRNA gene) and approx. $900 \mathrm{bp}$ of the LSU rRNA gene. The product of the first PCR served as template for a nested PCR amplifying an approx. 760 bp fragment targeting the LSU rRNA gene. A fusionprimer amplicon strategy was used. The forward primer LSU-D1f (5'-TAAGCGGAGGAAAAGAAAMTAAC-3') was synthesized together with the 454 adaptor $A$ and different multiplex identifiers (MIDs). The reverse primer LSUmBr (Krüger et al. 2009) was synthesized with the 454 adaptor B (Eurofins MWG Operon, Ebersberg, Germany). One $20 \mu \mathrm{l}$ PCR reaction contained $10 \mu \mathrm{l}$ of the Phusion High-Fidelity DNA Polymerase Mastermix (New England Biolabs, Frankfurt, Germany), $0.5 \mu \mathrm{M}$ of each primer and $0.2 \mu \mathrm{l}$ of the first PCR amplicon. The cycling conditions were $99^{\circ} \mathrm{C}$ for $5 \mathrm{~min}$, followed by $25 \times\left(99^{\circ} \mathrm{C}\right.$ for $15 \mathrm{~s}, 63{ }^{\circ} \mathrm{C}$ for $25 \mathrm{~s}, 72{ }^{\circ} \mathrm{C}$ for $35 \mathrm{~s}$ ), followed by $72{ }^{\circ} \mathrm{C}$ for $10 \mathrm{~min}$. For each sample, three individual PCRs were performed and the products were observed by gel electrophoresis. After confirming a visible band, PCR replicates were pooled.

The pooled samples were sent to the company IMGM Laboratories (Martinsried, Germany) where each amplicon was separately purified using solid phase reversible immobilization (SPRI) paramagnetic bead-based technology (Agencourt AMPure XP beads; Beckman Coulter, Krefeld, Germany) and quantified using PicoGreen dsDNA Assay Kit (Invitrogen, Darmstadt, Germany). Three libraries were generated containing the amplicon samples (pooled equimolarly) each with different MIDs. Each library was purified three times applying two different methods. First a gel extraction followed by a size selection step ( $>250 \mathrm{bp}$ ) performed twice using the SPRI paramagnetic beads. The DNA High Sensitivity LabChip Kit (Agilent Technologies, Waldbronn, Germany) was used on the 2100 Bioanalyzer (Agilent Technologies, Waldbronn, Germany) to measure the quality of the purified amplicon library. Sequencing was done by using the GS FLX+ Titanium Sequencing Kit (Roche, Basel, Switzerland).

The sequencing run has been stored at the Sequence Read Archive at the NCBI with accession number PRJNA242351.

\section{Bioinformatic analyses}

Image and signal raw pyrosequencing data were processed by the Roche 454 GS-FLX + inherent software packages applying the LongAmplicon3 processing pipeline which allows for 3 'end trimming, recommended for processing long amplicon reads. Further downstream analyses were carried out by using the QIIME pipeline (Caporaso et al. 2010). The following parameters were used to select reads: no more than 15 ambiguous bases, maximum length of homopolymer run of 15 , a maximum number of 5 primer mismatches and sequences with a minimum length of $500 \mathrm{bp}$ including the primers. Sequences which did not fulfill these requirements were discarded. Sets of sequences were divided into clusters using UCLUST (Edgar 2010) at a similarity threshold of $98 \%$. Representative sequences (RS) were used for downstream analyses either as OTUs or as input sequences for EPA based species affiliation. The $98 \%$ similarity threshold was empirically determined to always separate RS from different species into different clusters, by analyzing the approx. $760 \mathrm{bp}$ target region sequences from 1,167 well defined AMF reference sequences. After clustering, singletons were removed and the remaining RS were blasted against the NCBI database to identify and remove non-AMF sequences.

\section{Species delimitation}

Briefly, species delimitation consisted of two steps: in the first, a reference phylogenetic tree based on $1.5 \mathrm{~kb}$ sequences was computed and in the second, each RS of a $98 \%$ similarity cluster was individually placed into this reference tree and annotated to species.

For the first step, sequence alignments were done using MAFFT version 6 (Katoh et al. 2002) and manually optimized and merged with the reference alignment of Krüger et al. (2012) using ALIGN (www.sequentix.de) as described in Senés-Guerrero et al. (2014). A maximumlikelihood phylogenetic tree was calculated with RAxMLHPC2 at the CIPRES Science Gateway (http://www.phylo. org/portal2/) with 1,000 bootstraps and the GTRGAMMA model by using 1,078 unique $1.5 \mathrm{~kb}$ reference sequences from defined AMF species (Krüger et al. 2012) and from sequences obtained from a clone library constructed for Peruvian root samples (Senés-Guerrero et al. 2014). Additionally, new clone library (Krüger et al. 2009) sequencing data from Bolivia and Ecuador (accession numbers HG969311- 
HG969373; published here) were included. This reference phylogenetic tree was composed of only $1.5 \mathrm{~kb}$ sequences and used as a "phylogenetic backbone".

In the second step, each RS was aligned to the reference sequence alignment with MAFFT using a progressive method. The alignment containing the reference sequences plus the individual RS together with the reference tree were the input data for the phylogenetic placement of each RS. For this, the RAxML Evolutionary Placement Algorithm (EPA) with the GTRGAMMA model was used through the web interface (Berger et al. 2011; Berger and Stamatakis 2011). EPA individually assigns each sequence (in our case the RS) to the branches of the reference phylogenetic tree by using a maximum-likelihood model. The result consists of an interactive reference phylogenetic tree on which, by selecting tree branches, the affiliated sequences are displayed. A table with tree branches and affiliated sequences is also provided by EPA, which has been rigorously tested by its authors, proving its accuracy in placing short reads into a phylogenetic tree (for detailed information see Berger et al. 2011). Archaeopteryx Treeviewer 0.970 beta $\mathrm{X}$ was used to visualize the phylogenetic tree (Han and Zmasek 2009) and taxonomic annotations, following the most recent systematics of the Glomeromycota (Redecker et al. 2013), were manually done. Hence, each RS was affiliated to a described or yet unnamed species in the phylogenetic reference tree, as EPA also assigns "unknown" taxa to deeper branches in the tree which can be annotated at lower taxonomic levels.

To further analyze our species annotation method, we compared our previously published clone library Sangersequencing data obtained from Peruvian potato rhizosphere soil and root samples (Senés-Guerrero et al. 2014) with the 454 sequences obtained from the same root samples (rhizosphere soil was not included). To allow comparisons, species previously detected with the Sanger sequencing approach were annotated with the same species number in the present 454 sequencing analyses. 454-read relative abundance (RA) and the frequency of occurrence (FO) were the criteria to compare the samples.

\section{Validating taxa annotation: comparing $97 \%$-OTU, monophyletic clade and EPA based methods}

We compared two methods that are commonly used to delimitate AMF taxa against EPA, by using an artificially constructed AMF community sequence dataset. This was done to demonstrate the principle problems associated to the frequently used $97 \%$-OTU or simple monophyletic clade approaches. The artificial community consisted of 228 defined sequences comprising 7 closely related species from the genus Rhizophagus (see Krüger et al. 2012). Sequences from Rhizophagus irregularis were shortened to the same 760 bp LSU rDNA fragment as used in the 454-sequencing approach (from here onwards referred to as query sequences). These query sequences, derived from public database sequences, were annotated by three approaches: a $97 \%$ similarity threshold (97 \%-OTU), a monophyletic clade approach and species-affiliation using EPA.

Two maximum-likelihood phylogenetic trees were computed, one for the monophyletic clade and one for the EPA approach. For the $97 \%$ similarity threshold, the query sequences were clustered by using UCLUST and results displayed as OTUs in the tree computed for the monophyletic clade approach. For the monophyletic clade approach, the tree was computed from the $760 \mathrm{bp}$ target region only, to reproduce the strategy usually applied in pyrosequencing studies. For this, 228 Rhizophagus sequences, 176 query sequences and 14 Paraglomus sequences (as outgroup) were automatically aligned by using MAFFT and a maximum-likelihood phylogenetic tree was calculated using RAxML-HPC2 at the CIPRES Science Gateway with the GTRGAMMA model.

For the EPA approach the steps previously described were conducted. Briefly, a reference maximum-likelihood phylogenetic tree based on $1.5 \mathrm{~kb}$ sequences was calculated. The same sequences as used for the monophyletic clade approach were analyzed, except that some identical sequences were removed. The phylogenetic backbone reference tree contained 225 Rhizophagus sequences and 12 Paraglomus sequences. In the second step, 146 query sequences of approx. $760 \mathrm{bp}$ were individually placed into the branches of the reference tree by EPA and annotated to species.

With the intention to reproduce the methodology applied in many AMF ecological studies, OTUs obtained by a $97 \%$ similarity clustering were used with the artificially constructed Rhizophagus community at the validating step of the EPA-based method. For the analysis of the Andean samples, a $98 \%$ clustering step was used because this avoids merging different species in the same cluster (see Results section).

\section{Networks}

To display putative major AMF players associated with potato, two main sets of networks were analyzed and directly compared: OTUs (corresponding to $98 \%$ similarity threshold 454 representative sequences) and species (EPA based affiliation) networks. These sets of networks were created to visualize shared OTUs or species among different altitudes, plant developmental stages and plant varieties. Networks were produced following the QIIME pipeline and visualized using Cytoscape 3.0.1 (Cline et al. 2007). Dominant species were identified by analyzing RA (relative read abundance) and FO (frequency of occurrence). 


\section{Data analysis and statistics}

All analyses related to AMF community composition were performed using the vegan package (Oksanen et al. 2013) in R 3.1.0 (R Core Team 2014), unless stated otherwise.

To analyze and compare whether the method used to delimitate AMF taxa influences the interpretation of the community structure, OTU and species data matrixes were resampled to 200 reads and the Bray-Curtis index was used as a dissimilarity measure among the different sites. The pairwise distances from the sites in OTU and species datasets were compared. OTU and EPA derived data were analyzed by nonmetric multidimensional scaling (NMDS) using the metaMDS function with the BrayCurtis index. We used the envfit function to determine the relationship of altitude and plant stage with the OTUs and AMF species scores in the NMDS by using 999 permutations. Furthermore, changes on the beta-diversity were evaluated by permutational MANOVA (PerMANOVA) using the Arrhenius dissimilarity index with the adonis function. The dissimilarities for altitude and plant stage were partitioned and their significance analyzed by using 999 permutations. In this case, beta-diversity is used as a measure of heterogeneity and calculated taking into account the number of OTUs or species shared between two sites and the number of OTUs or species unique to each site. To exclude mixed effects in analyses of different altitudes and plant stages, we analyzed changes in beta-diversity of the plant stages at a similar altitude (approximately $3,500 \mathrm{~m}$; sites $\mathrm{B} 1, \mathrm{E} 4, \mathrm{P} 2$ ) and changes in altitude using samples from only the flowering stage. In addition, we tested the effect of altitude and plant stage within individual countries.

For both, the NMDS and beta-diversity analyses, two matrixes were the input. One matrix contained the normalized read counts of the OTUs or species found per sample and the second matrix contained the plant stage or altitude for each sample. Raw read counts were normalized using the package DESeq2 (Anders and Huber 2010) in R, as it was shown that other classical methods for read count normalization are not appropriate to detect differentially abundant species (McMurdie and Holmes 2014). To analyze altitude, we grouped the samples into 4 different altitude categories: $4=\geq$ $4,001 \mathrm{~m}, 3=3,561-4,000 \mathrm{~m}, 2=3,000-3,560 \mathrm{~m}$ and $1=<3$, $000 \mathrm{~m}$ (Online Resource 2).

\section{Results}

\section{Comparison among taxa annotation methods}

For our test dataset, based on publicly available validated sequences, the EPA approach resulted in all 146 R. irregularis
$760 \mathrm{bp}$ query sequences being correctly placed in the $R$. irregularis clade. None of the diverse sequences was misplaced into branches belonging to other Rhizophagus species (Fig. 1a and Online Resource 3). Thus, the correct result of the analysis was that all sequences belonged to one AMF species, $R$. irregularis.

When clustering the $R$. irregularis query sequences at a $97 \%$ similarity threshold, this resulted in 18 OTUs (Online Resource 4). Clustering the same sequences at $98 \%$ resulted in 32 OTUs (data not shown). The monophyletic clade approach resulted in a tree with very low bootstrap support values for many clades and it was difficult to delimit the different Rhizophagus species from one another (Figs. 1b-c, Online Resource 4). Even though the query sequences from $R$. irregularis were not clustering with sequences from other species, delimiting monophyletic clades to define species was not possible. To highlight the inaccuracy of taxa delimitation when using both, $97 \%$-OTUs and a monophyletic clade approach, we marked different sequences belonging to $97 \%$ OTUs in the tree computed for the monophyletic clade approach. Sequences from the same OTUs were spread over different monophyletic clades and one monophyletic clade could contain different OTUs (Fig. 1b, Online Resource 4). Furthermore, to indicate that intraspecific sequence variability leads to misinterpretation of phylotypes as different taxa or taxonomic units, we also labelled different sequence variants obtained from the well-studied culture $R$. irregularis DAOM197198 or from one single spore DNA extract of $R$. intraradices FL208 (Online Resource 4). The full reference phylogenetic tree used for the EPA approach and the placement of the query sequences are shown in the Online Resource 3. The full phylogenetic analysis of the monophyletic clade approach, also displaying the placement of $97 \%$-OTUs, is shown in the Online Resource 4.

\section{Processing of pyrosequencing data for AMF species affiliation}

From a total of 105 samples (12 samples each for Bolivia and Peru, 11 samples for Ecuador, from 4 altitudes and 3 plant developmental stages, in 3 replicates), PCR on 102 samples resulted in visible products. All three PCR-negative replicates were from the same sample (Peru, altitude 1, senescence stage) which was excluded in downstream analyses. The initial amount of reads was 698,297. After quality filtering and removing reads below $500 \mathrm{bp}, 366,088$ reads of at least $500 \mathrm{bp}$ length were clustered using a $98 \%$ similarity threshold into 4 , 943 representative sequences (RS). This was done because for the region analyzed, the frequently used similarity threshold of $97 \%$ led to a number of clusters containing sequences from different species (e.g., sequences of Gigaspora rosea with G. margarita, Funneliformis mosseae with F. coronatus, Claroideoglomus luteum with $C$. claroideum and 


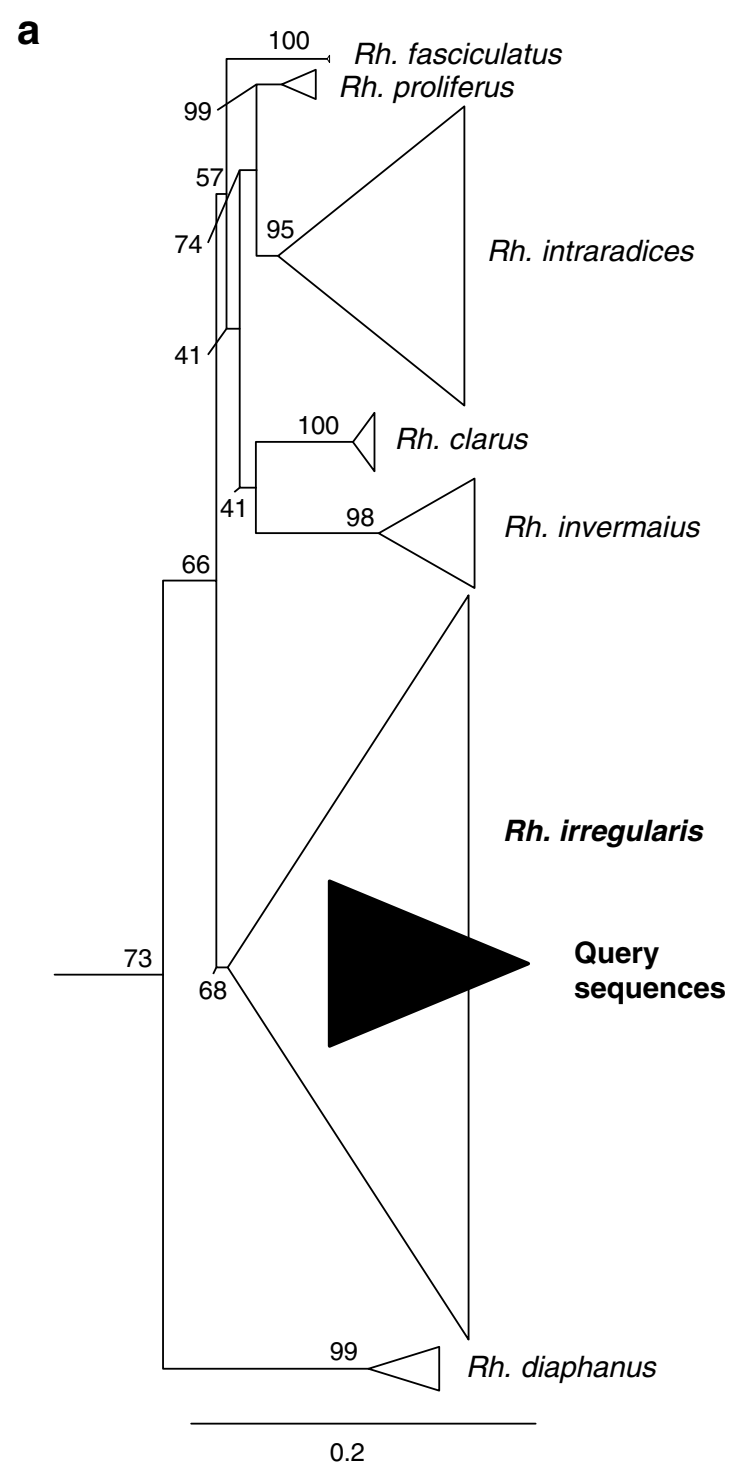

Fig. 1 Maximum-likelihood phylogenetic trees showing different AMF taxa delimitation methods. Sequences from $R$. irregularis were used as query sequences and were annotated to species in the genus Rhizophagus by using EPA (a), clustering at $97 \%$ similarity threshold (97\%-OTUs) (b), and a monophyletic clade approach (b and c). $97 \%$-OTUs are marked in blue and sequences used as query sequences in red. Reference sequences are in black. Using EPA species affiliation, all

Acaulospura scrobiculata with A. spinosa; data not shown). This problem could be avoided by using a $98 \%$ similarity threshold for clustering.

After singleton removal, 3,218 RS were left, of which 956 $(29.7 \%)$ were non-AMF. From these, $96 \%$ were from fungi, $3.6 \%$ from plants and $0.4 \%$ from protozoa. Finally, 2,262 RS containing 255,740 reads with an average read length of 718 bp remained for AMF. By using EPA we annotated these to 41 species from 12 AMF genera (Figs. 2 and 3; for read abundance see Online Resource 5).

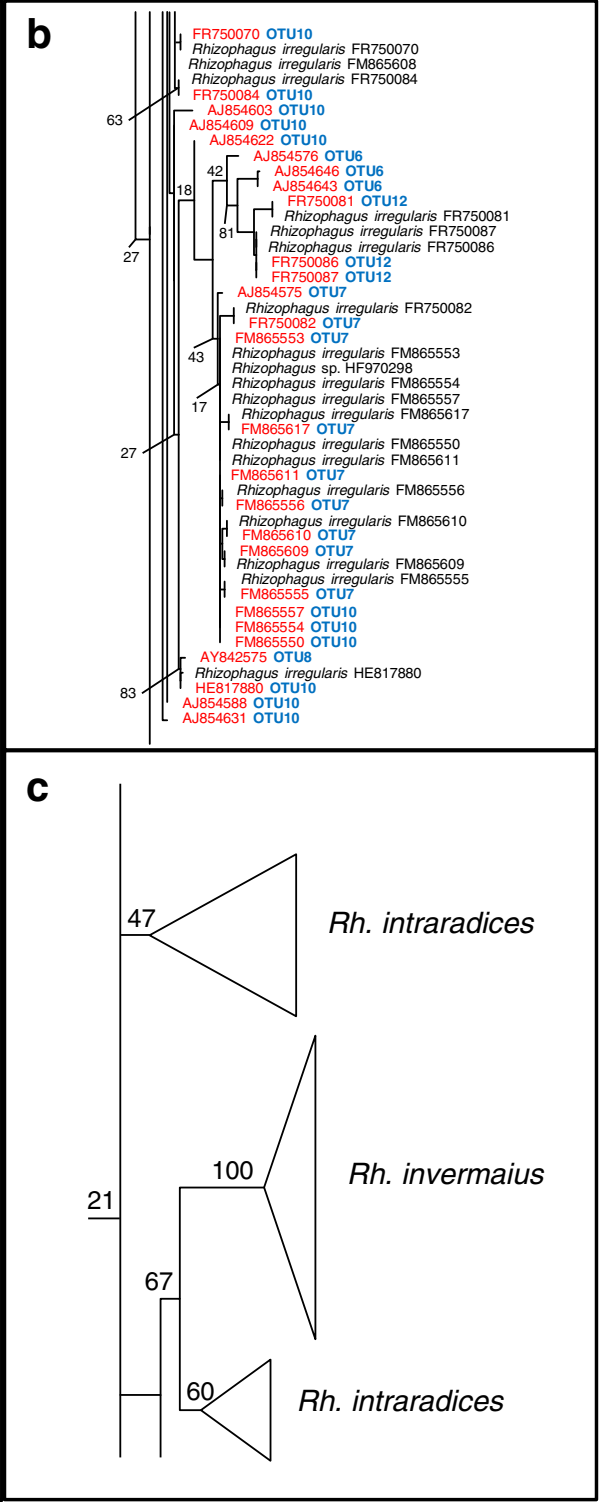

query sequences were correctly placed in the R. irregularis clade (a). Problems when annotating 454 reads by using $97 \%$-OTUs are shown: two OTUs in a single monophyletic clade (OTU6 and 12), spread OTUs partly clustering with other OTUs (OTU10) (b). Problems when annotating species using $\sim 760$ bp sequences and a monophyletic clade approach are shown: low phylogenetic resolution not allowing species definition (c)

\section{Comparing OTUs against EPA based species affiliation for potato associated AMF}

To demonstrate how the annotation of OTUs or species can influence our understanding of the AMF community composition, we used the identical dataset to visualize networks containing either OTUs or species. The OTU networks always showed a similar trend in which the majority of the OTUs was specific to one variable (one altitude, plant stage or potato variety). On the contrary, 


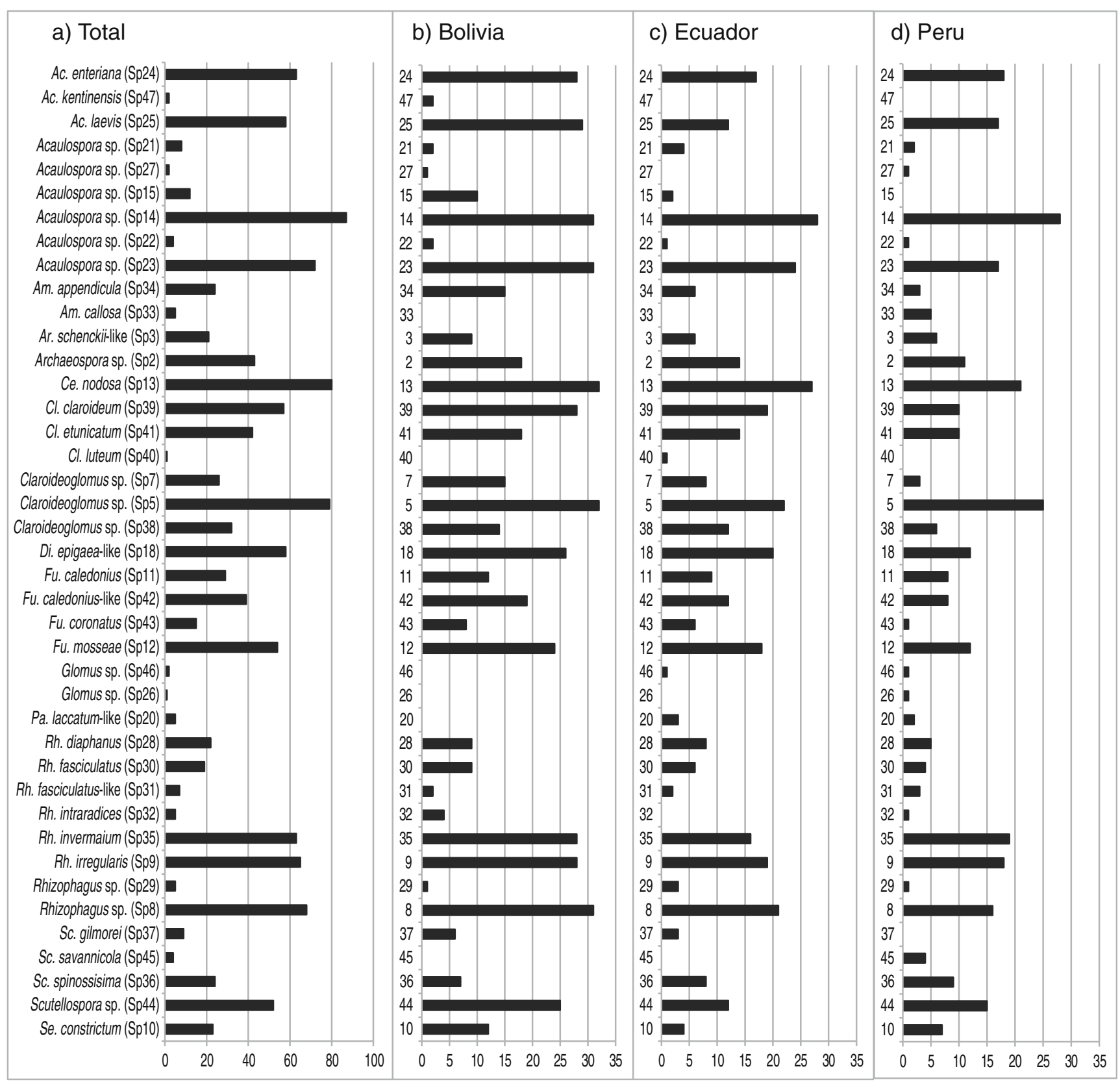

No. of plant replicates

Fig. 2 Frequency of occurrence (FO) of 41 annotated AMF species and the number of individual root samples (total root samples analyzed $=102$ ) in which they were found. A conserved species pattern can be observed in

species networks indicated that most of the AMF species were shared and only few were specific to a certain condition (Fig. 4).

Furthermore, we analyzed the similarities of the AMF communities among the sampling sites for both, OTUs and species by using the Bray-Curtis index. For comparison, we did a sub-sampling step; we excluded the samples E3Sc and E4Sc from the species data and E1Sc and E2Sc from the OTUs data because of having fewer than 200 reads. The Bray-Curtis similarity for OTUs indicated the three countries. The same conserved patterns can be observed when analyzing different altitudes or plant stages shown in Online Resource 7

a community composition represented by two major clusters (Fig. 5a) whereas when analyzing species, the AMF community composition of the sites appeared less structured (Fig. 5b). Importantly, the clustering topology showed that the OTU-defined community composition is totally inconsistent with the species-defined community (Fig. 5). As EPA species affiliation must be interpreted more robust than $97 \%$-OTU and also basic monophyletic clade approaches, the community composition based on OTUs analyses appears unreliable. 


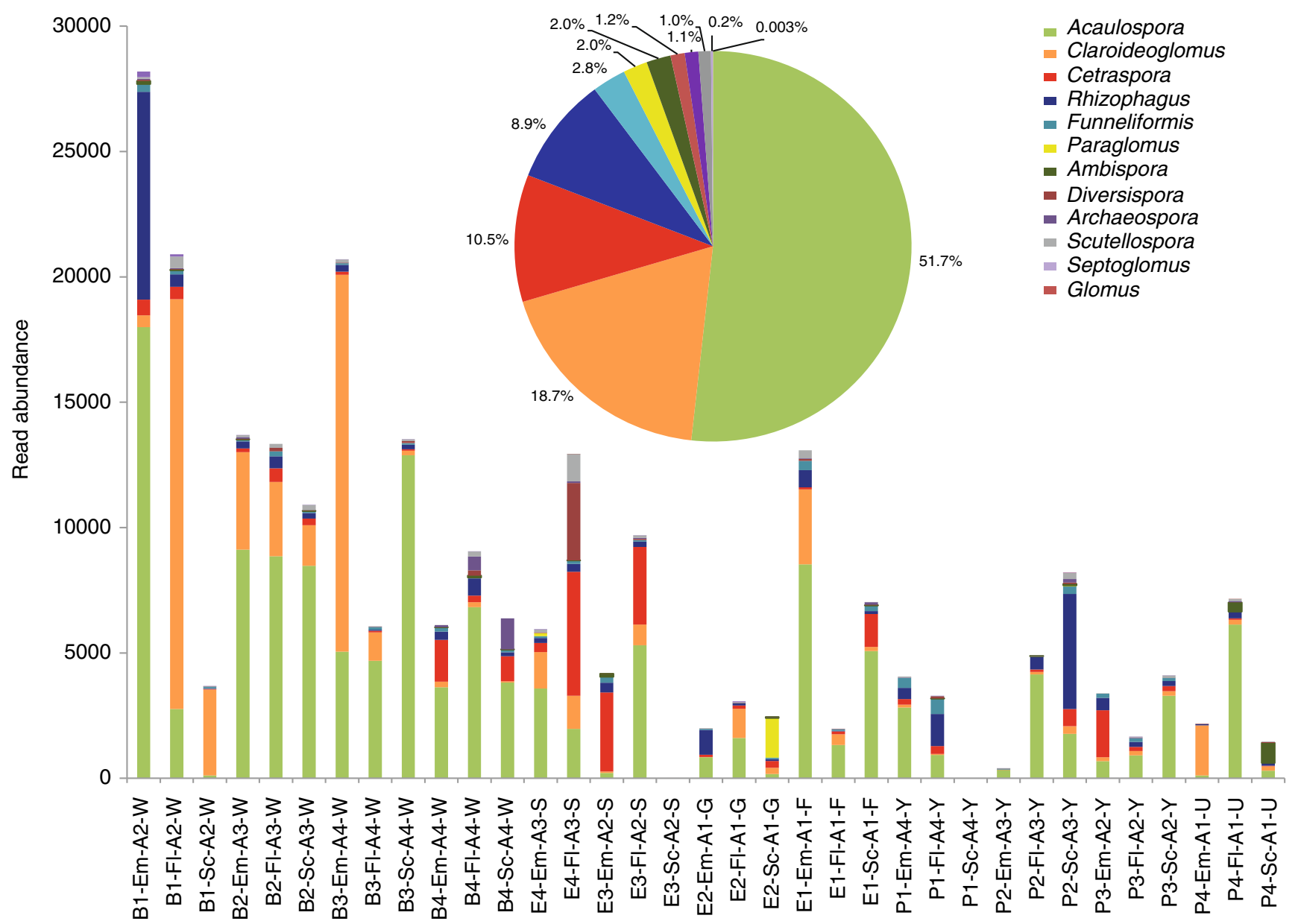

Fig. 3 454-read abundance of the 12 AMF genera found at 12 field sites $(\mathrm{B}=$ Bolivia, $\mathrm{E}=$ Ecuador, $\mathrm{P}=\mathrm{Peru} ; 1=$ site $1,2=$ site $2,3=$ site $3,4=$ site 4 ; $\mathrm{Em}=$ emergence, $\mathrm{Fl}=$ flowering, $\mathrm{Sc}=$ senescence; $\mathrm{A} 1=$ altitude group 1 $(<3,000 \mathrm{~m}), \mathrm{A} 2=$ altitude group $2(3,000-3,560 \mathrm{~m}), \mathrm{A} 3=$ altitude group

\section{AMF species diversity in potato roots}

We demonstrated that the EPA annotation of species is more robust compared to other frequently used methods and that conclusions on the community composition would strongly rely on the method used for taxa definition. We therefore, based on published knowledge (Stockinger et al. 2010) and our empirical data, analyzed EPA based species-affiliation data for further interpretation.

Rarefaction curves showed that plateau levels were reached for most of the samples when analyzing at the species level (Online Resource 5b). Based on relative read abundance (RA), the five most abundant species (from lowest to highest) were an unknown Claroideoglomus sp. (Sp. 5), Claroideoglomus claroideum (Sp. 39), Cetraspora nodosa (syn. Scutellospora nodosa) (Sp. 13), an unknown Acaulospora sp. (Sp. 23), and another unknown Acaulospora sp. (Sp. 14) (Online Resource 6). Based on frequency of occurrence (FO), the vast majority of potato plants ( $85 \%, 87$ plants) were colonized by the most abundant Acaulospora Sp. 14 (Fig. 2 and Online Resource 7).
$3(3,561-4,000 \mathrm{~m}), \mathrm{A} 4=$ altitude group $4(\geq 4,001 \mathrm{~m}) ; \mathrm{W}=$ Waycha, $\mathrm{S}=$ Superchola, $\mathrm{G}=$ Guata, $\mathrm{F}=$ Fripapa, $\mathrm{Y}=$ Yungay, $\mathrm{U}=$ Unica. Inset shows the relative abundance in percentage for each genus

\section{Preferential potato root colonizers}

We considered that the species colonizing the plants at most altitudes, plant stages and potato varieties, were preferential colonizers of potato, based on FO. No species was present in all of the samples, nevertheless the overall most abundant (based on RA) unknown Acaulospora sp. (Sp. 14) appeared

Fig. 4 Networks showing the distribution of AMF OTUs and species, based on the identical representative sequences (RS) of $98 \%$ similarity clusters. Nodes correspond to either OTUs or species and connecting edges indicate different conditions. OTU (a) and species (b) networks at different altitude ranges; 1 (green $)=$ altitude $1(<3,000 \mathrm{~m}), 2($ blue $)=$ altitude $2(3,000-3,560 \mathrm{~m}), 3$ (purple) $=$ altitude $3(3,561-4,000 \mathrm{~m}), 4$ (orange) $=$ altitude $4(\geq 4,001 \mathrm{~m})$. c) OTU and d) species networks of AMF colonizing different plant stages; $\mathrm{Em}=$ emergence (green), $\mathrm{Fl}=$ flowering (orange), $\mathrm{Sc}=$ senescence (purple). e) OTU and f) species networks of AMF colonizing different potato varieties; $\mathrm{W}=$ Waycha (orange), $\mathrm{U}=$ Unica (violet), $\mathrm{F}=$ Fripapa (green), $\mathrm{G}=\mathrm{Guata}($ blue), $\mathrm{S}=$ Superchola (purple), Y=Yungay (red). In the species networks, nodes show the species number and nodes highlighted in yellow represent species shared by all the different variables 


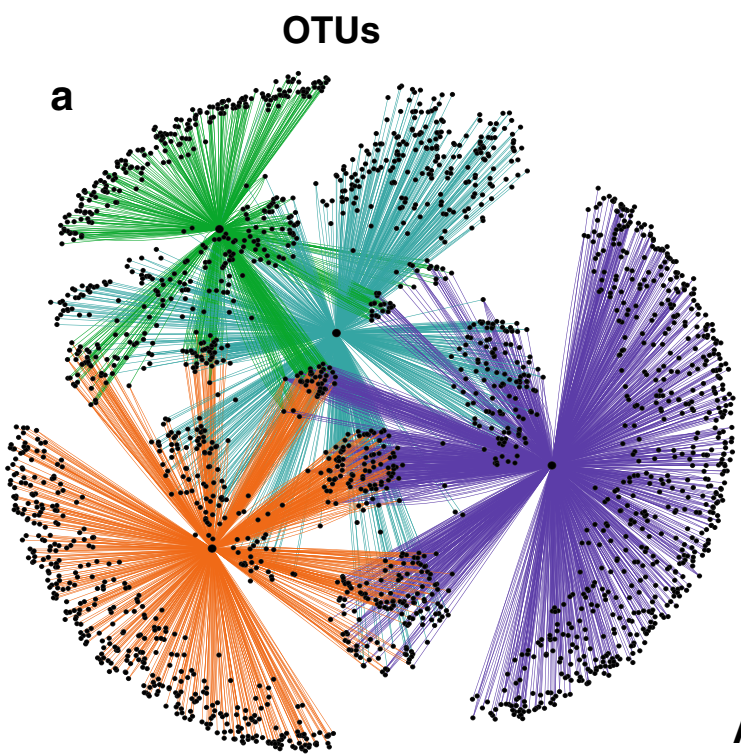

Altitude

\section{Species}

b

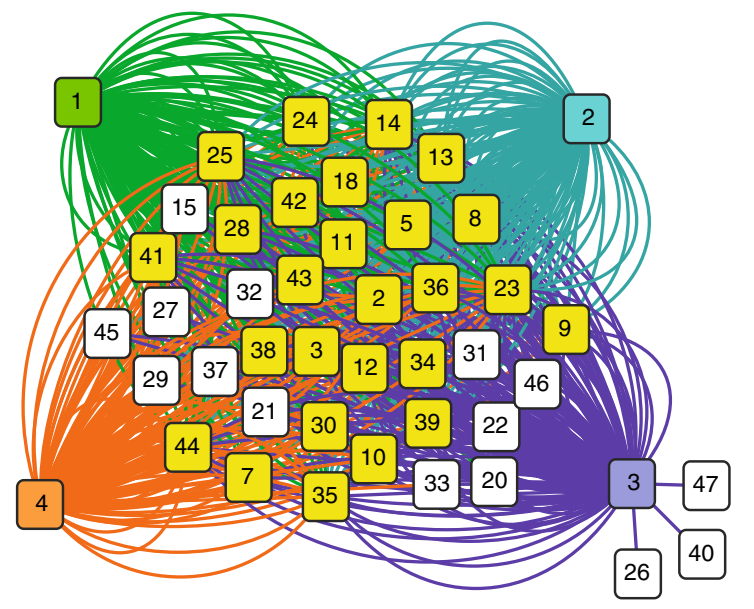

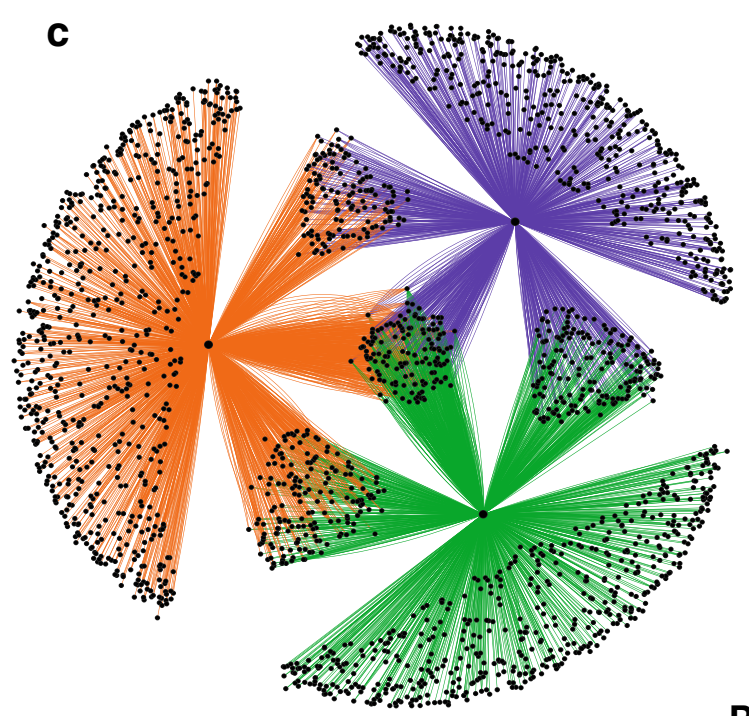

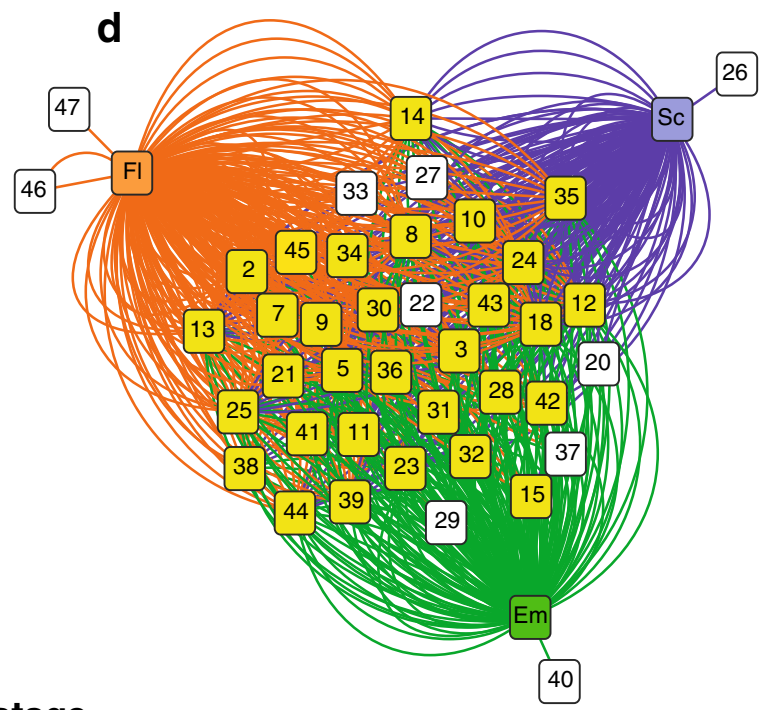

Plant stage

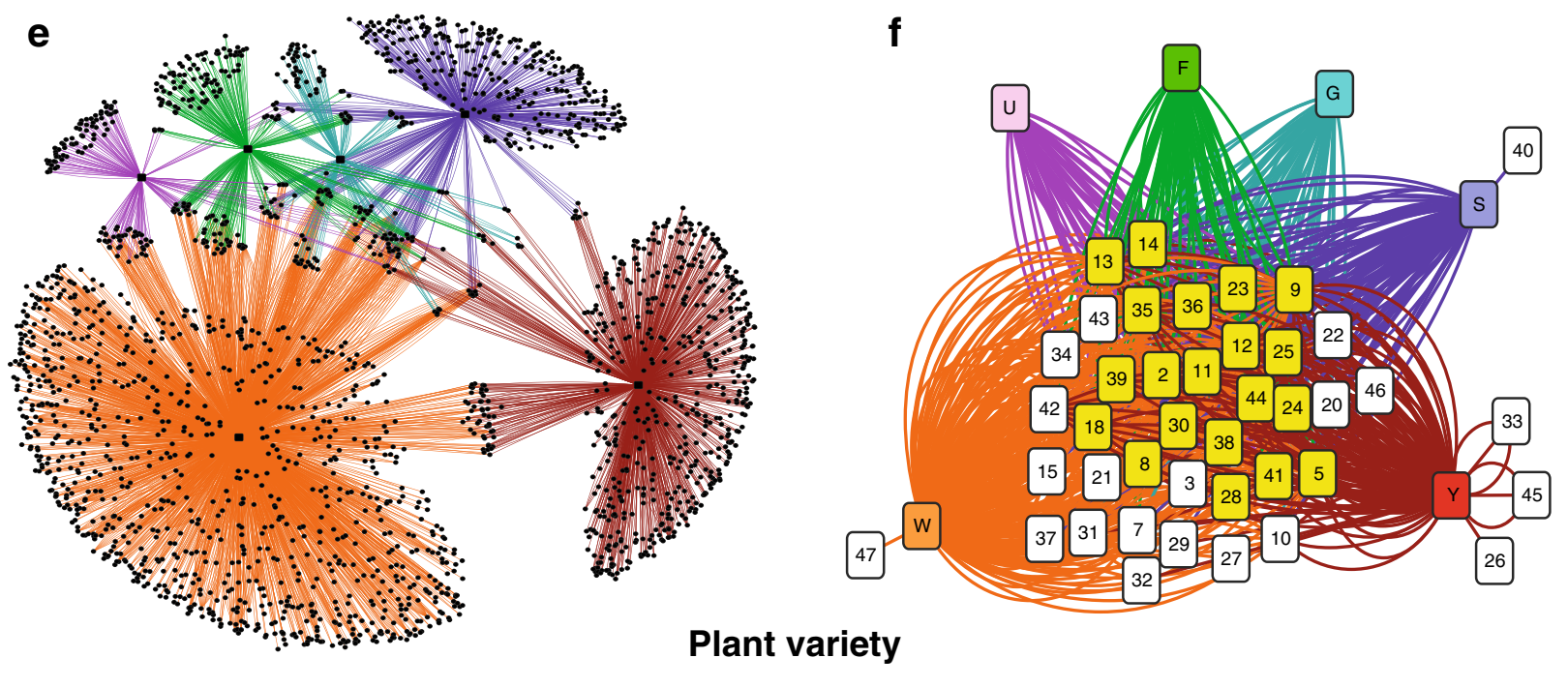


a

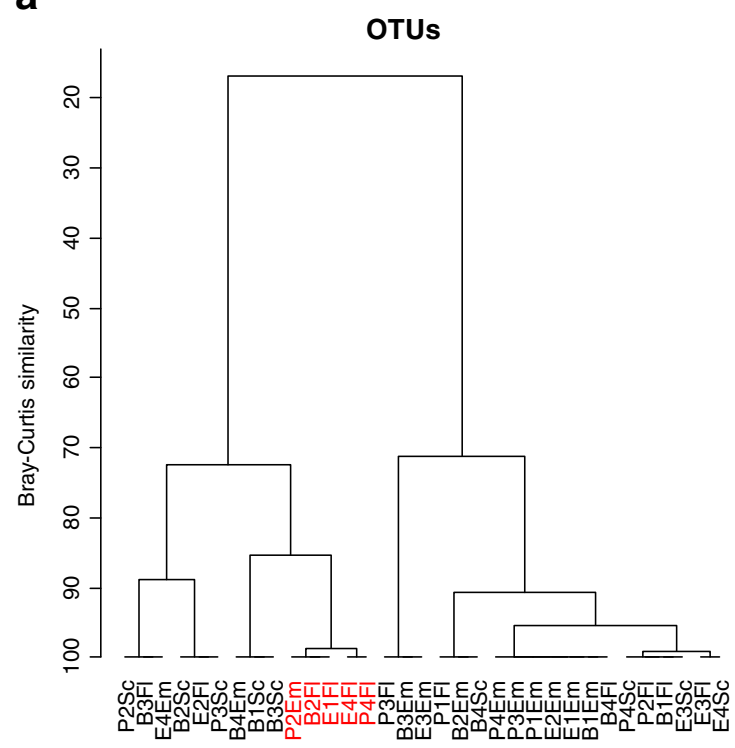

Fig. 5 Bray-Curtis similarity of the sampled sites when using OTUs (a) or species (b) to delimit the AMF community. Example sites that are identical when analyzing OTUs and different when analyzing species

in $85 \%$ of the samples, followed by C. nodosa (Sp. 13; 3rd most abundant species based on RA) and Claroideoglomus sp. (Sp. 5; 5th most abundant species) each appearing in $78 \%$ of the samples (see Fig. 2 for FO values and Online Resource 6 for RA values). Acaulospora sp. (Sp. 23; 2nd most abundant species) appeared in $71 \%$ and C. claroideum (Sp. 39; 4th most abundant species) appeared in $56 \%$ of the samples. These species therefore can be interpreted as a conserved core-species community of potato in the Andes.

Regarding coexistence of species, from 87 plants that hosted Acaulospora sp. (Sp. 14), 75 plants (86\%) also contained C. nodosa (Sp. 13) and a Claroideoglomus sp. (Sp. 5). Regarding genera, Cetraspora, Rhizophagus, Acaulospora and Claroideoglomus colonized 68 plants (67\%) simultaneously.

Among the six different potato varieties, 20 (49\%) from the 41 total AMF species are shared among all of them. For the plant developmental stages, 31 species (76\%) are shared among the three stages. For altitude, 26 species $(63 \%)$ are shared among the four altitude groups (Fig. 4 and Online Resource 7).

\section{Number of species colonizing an individual potato root system}

The AMF species number in a single root system was displayed as different size groups (zero species, 1 to 5 species, 6 to 10 species, etc.; Fig. 6). 102 individual root systems contained 1 to 25 species in relatively similar amounts. Two percent of the root samples contained more than 25 species (Fig. 6). The amount of species found in a single root sample b

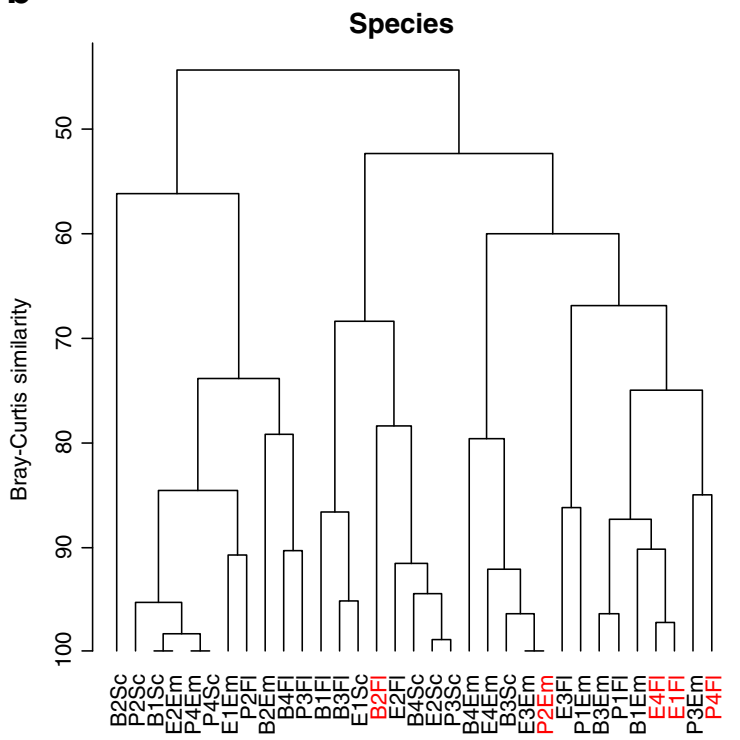

are highlighted in red. $\mathrm{B}=$ Bolivia, $\mathrm{E}=$ Ecuador, $\mathrm{P}=\mathrm{Peru} ; \mathrm{1}=$ site $1,2=$ site 2, 3=site 3, 4=site 4; Em=emergence, $\mathrm{Fl}=$ flowering, $\mathrm{Sc}=$ senescence

was not related to the plant stage, e.g., for some plants in the emergence stage more than 20 species could be detected while for others only less than five.

\section{Influence of altitude and plant stage on AMF communities}

Nonmetric multidimensional scaling analysis of the species data resulted in a two-dimensional solution with a total stress value of 0.2 for both OTUs and species. The OTU NMDS plot revealed tight clusters of OTUs separated from other clusters

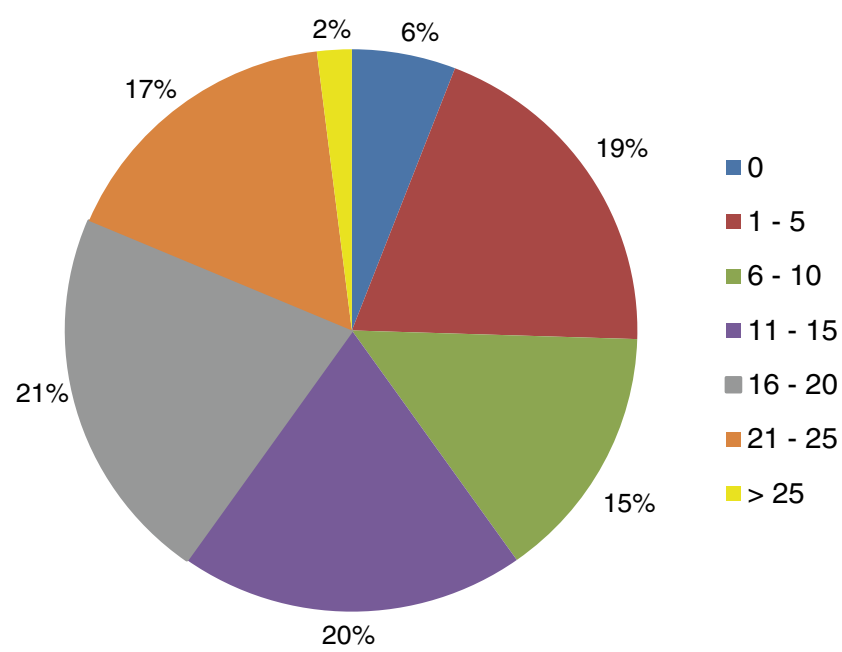

Fig. 6 Number of AMF species, in percentage, detected in individual root system samples. Zero to more than 25 species were separated in seven size groups $(0,1$ to 5,6 to 10,11 to 15,16 to 20,21 to $25,>25$ species). The percentage of AMF species found (in a single root system) within these groups is shown 
Fig. 7 Nonmetric multidimensional scaling (NMDS) ordination plot for OTUs (a) and the $41 \mathrm{AMF}$ annotated species, color-coded by their genus (b). Ellipses represent $95 \%$ confidence intervals around the four altitude groups and the three plant stages centroids. Nonoverlapping centroids are considered significantly different at $\alpha=0.05$. Colors of ellipses: green $=$ altitude $1(<3,000 \mathrm{~m})$, blue $=$ altitude $2(3,000-3,560 \mathrm{~m})$, purple $=$ altitude $3(3,561-$ $4,000 \mathrm{~m})$, orange $=$ altitude 4 $(\geq 4,001 \mathrm{~m})$, red $=$ emergence, gray $=$ flowering, black $=$ senescence a

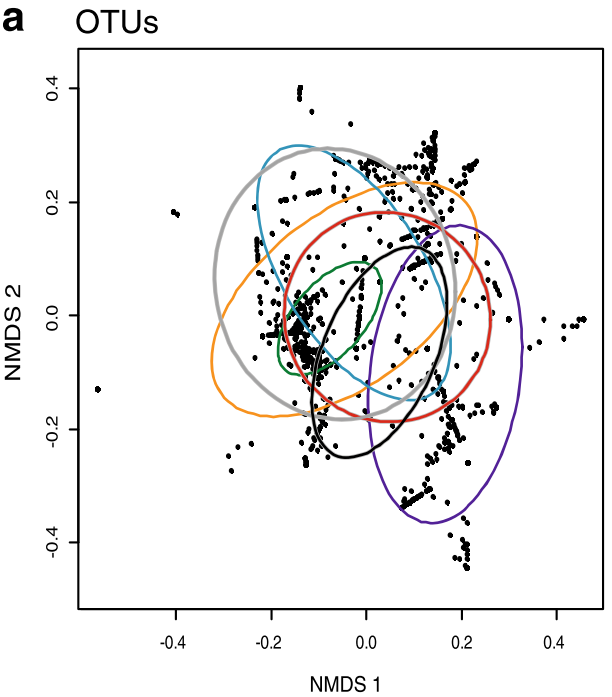

b Species

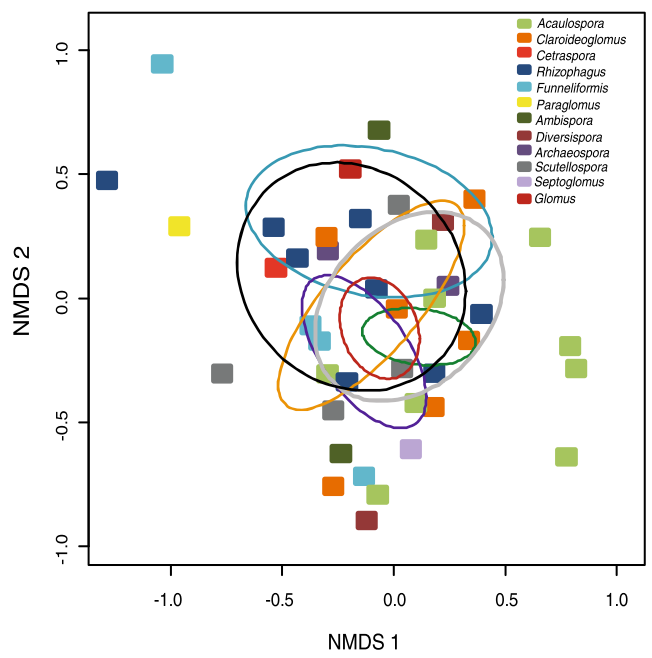

which may indicate sub-populations in the data (Fig. 7a). The species NMDS revealed no structure in the community composition when we marked the 41 species based on their genus (Fig. 7b). Moreover, altitude and plant stage had no significant influence on the AMF community based on OTUs or species (Table 1) and this did not change when increasing the NMDS dimensions (data not shown). However, the PerMANOVA conducted to test whether altitude or plant stage were influencing the beta-diversity of the different sites showed that altitude was a significant factor $(P<0.05)$ in both datasets, with the effect being stronger for the OTUs (Online Resource 8). PerMANOVA on the beta-diversity conducted on different sites at the same plant stage revealed that altitude may be a significant factor $(P$-value $=0.052)$ whereas the same test conducted on sites at a similar altitude revealed that the influence of plant stage was not significant (Online Resource 9). Doing the same test for individual countries, using both OTU and species data, neither altitude nor plant stage were significantly affecting the community composition, with the exception of OTU data for Peru (Online Resource 10).

Table 1 Squared correlation coefficients $\left(\mathrm{R}^{2}\right)$ for the centroids in the NMDS ordination performed with normalized read counts for the OTUs and species data

\begin{tabular}{llll}
\hline & Environmental variable & $\mathrm{R}^{2}$ & $P$ \\
\hline OTUs & Altitude & 0.1515 & 0.116 \\
& Plant stage & 0.0561 & 0.477 \\
Species & Altitude & 0.1658 & 0.074 \\
& Plant stage & 0.0658 & 0.379 \\
\hline
\end{tabular}

$P$-values are based on 999 permutations

\section{Comparison with a clone library Sanger sequencing approach}

From clone libraries derived from Peruvian samples (24 root and 12 pooled rhizosphere soil samples) we annotated 20 species, some of them found only in rhizosphere soil in a previous study (Senés-Guerrero et al. 2014) indicating a probable number of $31 \pm 8$ species by the Chao index. From the 454 sequences derived from the same root samples of Peru (35 samples) we annotated 37 species, including 10 species (out of 12) found in the root samples of the clone library. An Ambispora sp. and an Archaeospora sp. found in roots and previously annotated in the clone library as "Sp. 1" and "Sp. 4 " were not found by 454 sequencing. Other species $(6,16,17$ and 19) that were found only in rhizosphere soil with the Sanger sequencing approach were also not detected by 454 sequencing of the root samples.

\section{Discussion}

\section{Methods for AMF species delimitation}

Here, for the first time, we used 454 GS-FLX+ pyrosequencing of an approx. $760 \mathrm{bp} \mathrm{LSU}$ rDNA amplicon together with a high throughput, maximum-likelihood based phylogenetic annotation approach (EPA) to monitor AMF in the field at species level. This approach is based on an aligned reference sequence database and a maximum-likelihood phylogenetic reference tree. We used EPA because it provides more accurate species annotation than other methods used for high throughput monitoring and community studies of AMF, considering theoretical assumptions, published data (Stockinger 
et al. 2010; Berger and Stamatakis 2011) and our empirical tests. Next generation sequencing technologies are advancing rapidly and for fungi, a metabarcoding pipeline using the paired end $(2 \times 250 \mathrm{bp})$ Illumina MiSeq platform has been developed (Bálint et al. 2014). Similar methods could be employed for AMF once longer read lengths could be obtained, providing sufficient phylogenetic signal to distinguish closely related species.

In general, pyrosequencing data analysis pipelines can have a strong impact on the biological conclusions (Bakker et al. 2012). Using a precise method is crucial to obtain ecologically meaningful data and for fungi some specific considerations have been highlighted (Lindahl et al. 2013). For AMF, the difficulty starts from selecting the genetic marker region, continues with the similarity threshold used to cluster sequence reads and increases when trying to assign sequences into taxonomic groups. Moreover, every type of analysis depends on the quality of the baseline data used to compare with. In this context one problem for AMF (and also Fungi in general) is, that many species have not yet been described or characterized by DNA sequences, which is especially problematic in unexplored ecosystems harboring high diversity. In such regions, many unknown AMF species are expected to exist (Kivlin et al. 2011; Liu et al. 2011), as shown for the Andean region studied here with about $50 \%$ of the AMF species detected being uncharacterized by DNA sequences (Senés-Guerrero et al. 2014).

It is not possible to discriminate closely related AMF species by using simple sequence similarity thresholds and/or phylogenetic cluster analyses based on limited phylogenetic signal, for example when analyzing short average reads lengths of 200-400 bp as obtained with the previous 454 GS-FLX technologies. Thus, until now, a robust tracing of AMF at the species level has not been possible with 454 sequencing, but data regarding basic AMF community patterns and the putative factors driving such communities have been obtained (Öpik et al. 2009; Dumbrell et al. 2011; Davison et al. 2012; Lekberg et al. 2012; Öpik et al. 2013). Most of the earlier 454 sequencing based AMF community studies analyzed data by clustering reads into $97 \%$-OTUs and matching them using BLAST. Some of the drawbacks of this approach are that many fungal sequences in public databases have not been correctly annotated or have quality issues (Kõljalg et al. 2013), leading to incorrect species descriptions and also, that whenever a taxon is not present in the reference sequences, the BLAST based assignment can be misleading (Berger et al. 2011). Another approach for the analysis of 454 sequencing data is the definition of OTUs based on clades in phylogenetic trees including known sequences (Horn et al. 2014). The latter approach is called a monophyletic clade approach and may, besides the formation of clades, also use the support values for the respective clades to interpret OTUs. Nevertheless, calculating a phylogenetic tree using short read length sequences, even of $760 \mathrm{bp}$ lengths, leads to low resolution and support for clades and is prone to misinterpretations (see Online Resource 4). Lekberg et al. (2014) suggested that defining OTUs by using either a similarity threshold of $97 \%$ or by using a monophyletic clade approach did not affect interpretations of the general AMF community patterns. However, this assumption depends on the level of interpretation. Using a monophyletic clade approach based on short sequences does not provide species resolution and the taxonomic level of OTUs is undefined (see Online Resource 4). The high intraspecific variability of the rDNA sequences of AMF may cause the consequent splitting of individual species into many OTUs when using \%-thresholds, as well when using monophyletic clades. Beside the noise of such data, this may cause OTU undersampling and consequent misinterpretations. Another issue that affects the interpretation of sequencing data occurs at the clustering step, where greedy heuristic clustering can result in incorrect grouping of sequences into clusters (Sun et al. 2012) leading to misinterpretations of taxa abundance and especially affecting the significance of rare taxa.

Thus, when circumscribing 454 sequences to AMF taxonomic units many factors, which are unfortunately often ignored, can lead to misinterpretations, including primer selection, BLAST-based annotation, similarity thresholds, method of OTU definition, and ecosystems with many unknown species. We tried to reduce the afore mentioned problems by i) selecting a region that previously was discussed to provide species resolution when using 454-sequencing (Stockinger et al. 2010), ii) testing similarity thresholds for sequence clustering using reference sequences as an empiric dataset to avoid sequences from different species falling into the same cluster and iii) using an EPA based approach for species annotation, in which sequences are individually placed into a phylogenetic reference tree. Even though such annotation may also be biased (e.g., due to misalignment of sequences, see Berger and Stamatakis 2011), it appears to be significantly better for AMF community structure analyses than any of the other commonly used methods for high throughput sequencing data analysis. We could robustly annotate the representative sequences to unknown as well as to described species.

\section{Taxonomic coverage and species diversity}

Interpretations of AMF diversity are strongly influenced by the PCR primer choice. Some primer combinations discriminate against certain AMF lineages (Gamper et al. 2009), while others result in high non-specific amplification (Alguacil et al. 2009). The region that we amplified in the first PCR offers species resolution power (Stockinger et al. 2010) and the primers used (Krüger et al. 2009) allow the widest taxon coverage compared to other commonly used primers targeting a single nuclear rDNA marker (Kohout et al. 2014). It may be mentioned that Kohout et al. (2014) reported chimera 
formation when using these primers. However, this was interpreted based on the use of Taq DNA polymerase, whereas the use of a high-fidelity enzyme with a fused DNA-binding domain, as the Phusion DNA polymerase recommended by Krüger et al. (2009), prevents many of the processes leading to chimera formation.

Here we annotated 41 species, 15 of them (37\%) unknown or previously not described in sequence databases and 5 of them (12\%) closely related to but separated at the species level from known species. Other studies using 454 sequencing have reported 70 AMF OTUs in an area of approximately $7 \mathrm{~m}^{2}$, analyzing samples at summer and winter seasons (Dumbrell et al. 2011), 32 AMF OTUs in grassland plots (Lekberg et al. 2012), and 37 (Davison et al. 2012) and 48 (Öpik et al. 2009) AMF OTUs in forest plots. However, interpreting and comparing richness and species diversity is difficult from afore mentioned studies, for example because the number of OTUs is strongly dependent on the variability of the marker region and the threshold value used. We demonstrated this by the analysis of published LSU sequences of $R$. irregularis, resulting in 18 OTUs at $97 \%$ or 32 OTUs at $98 \%$ similarity, for one species only (see Online Resource 4).

\section{AMF species associated with potato}

To determine AMF preferentially colonizing potato, we used proportional read abundance as a semi-quantitative measure of abundance. Despite known biases when using 454 reads to determine biological abundance (e.g., rRNA gene copy numbers, primer bias, varying PCR efficiency in different samples), comparing proportional abundance of one species with itself across samples and replicate 454 runs is reliable (Amend et al. 2010; Kauserud et al. 2012). Interestingly our data show that most of the samples are colonized by a conserved group of AMF species ( $67 \%$ of the plants from 12 studied sites were colonized by certain species from the genera Acaulospora, Cetraspora, Claroideoglomus and Rhizophagus simultaneously) which appear to be main players in potato AM in the Andean region. For the potato plants it is both surprising and noticeable that this core AMF species community is relatively conserved throughout the studied region since the sampling sites are from remote areas, e.g., a distance of 3,169 km from Loja (Ecuador) to Cochabamba (Bolivia), and from a wide variety of climatic conditions, e.g., in Cochabamba the average day temperature is approx. $24^{\circ} \mathrm{C}$ during summer and mild during winter, whereas at study sites above 4,000 mamsl even in summer frost may occur periodically at night and strong frosts are frequent during winter.

In previous studies, F. mosseae was the only species found colonizing potato roots in soil-trap cultures (Bharadwaj et al. 2007) and R. irregularis (as "Glomus intraradices") was reported as the preferential colonizer of potatoes in an Italian agricultural field at low altitude ( 85 mamsl) (Cesaro et al.
2008). In the Andean region, surprisingly the potato roots colonizers detected in highest relative abundance and frequency were two unknown Acaulospora spp. (Sp. 14 and Sp. 23) found individually or together at all conditions and in 91 ( $89 \%$ ) of the samples analyzed, perhaps indicating host preferences. The very frequent appearance of Acaulospora spp. in the Andes might directly or indirectly be related to altitude, since members of this genus were also frequently reported at altitudes around 3,000 $\mathrm{m}$ in the Alps and in the Chilean Andes (Oehl et al. 2006, 2011), as well as at up to $3,520 \mathrm{~m}$ in the South American Puna grassland (Lugo et al. 2008) and at the Tibetan Plateau (Li et al. 2014; Gai et al. 2012). Due to their high abundance (RA) and frequency of occurrence (FO), we considered these Acaulospora spp. as main potato colonizers in the Andes. We cannot yet state about the biogeography of these species because of insufficient availability of data for comparison.

\section{AMF species diversity in individual potato root systems}

An individual plant root system is usually colonized by several AMF species. For potato plants high levels of AMF colonization were reported (McArthur and Knowles 1992; Davies et al. 2005) but surprisingly only one or two species per root system were detected by Sanger sequencing of clone libraries from Peruvian ecosystems (Senés-Guerrero et al. 2014). In contrast, 454 sequencing from the same samples as analyzed in that study revealed that more than 25 AMF species can be present in a single root system, indicating the limitations of the clone library based approach. The joint presence of certain species may be related to phylogenetically distinct AMF having different complementarity functions (Maherali and Klironomos 2007). For potato, the frequency of occurrence of AMF in individual root systems strongly indicates functional complementarity beneficial for the host, based on the observation that an Acaulospora sp. (Sp. 14), C. nodosa (Sp. 13), and a Claroideoglomus sp. (Sp. 5) were found coexisting in $74 \%$ of all samples (75 plants). Sixty seven percent of all samples (68 plants) additionally contained one of the eight detected Rhizophagus species.

\section{Influence of plant stage and altitude on AMF species communities}

Analyzes of OTU networks indicate that most of the OTUs are specific to either an altitude, plant stage or plant variety, in agreement with previous studies showing habitat or seasonal differences and host preferences in the AMF communities (Husband et al. 2002; Öpik et al. 2009; Dumbrell et al. 2011; Kivlin et al. 2011). Contrary, the EPA-based species networks based on the same data showed that most of the AMF species appeared at all altitudes, plant stages and plant varieties. Also when using the Bray-Curtis index the results 
obtained for OTU and species datasets were contradictory. Bray-Curtis similarities for AMF communities were in general higher when analyzed by their OTUs than when analyzed as species, but most important is that the analysis of the OTUs resulted in a completely different topology of clustering compared to that of the species. A higher similarity of OTU clusters was also observed in the NMDS plot.

Because the EPA based species affiliation method must be considered as much superior to the $98 \%$ or $97 \%$ similaritybased OTU affiliation, we conclude that results derived from such OTUs must be interpreted with caution.

The contradictory results among OTUs and species can partly be explained by different OTUs being detected in different samples, but representing the same species. Such OTUs seem to be unique for that sample, yet, this uniqueness may in many cases be based on undersampled highly variable DNA phylotypes.

Surprisingly, even though OTU and species communities appear different, plant stages or altitude showed no significant influence on the AMF community for both datasets as analyzed in the NMDS plots. However, the beta-diversity of the sites was significantly influenced by altitude according to the perMANOVA analysis. Because the analyses are different, the outcomes should be interpreted in different ways. In NMDS, the variation is reduced to an ordination space and the environmental variables are fitted to the ordination scores. The beta-diversity is analyzed by the number of taxa that are shared or are unique among sites. As many OTUs represent the same species, in the case of OTUs the response to altitude or plant stage is stronger. This may be due to some highly abundant and frequent AMF and noisy data for rare species, which are represented as "randomly" occurring OTU variants. Even if we found that altitude is a significant factor when we analyzed different sites for the same plant stage, we cannot exclude that the influence of altitude is due to other factors found between sites, as altitude was not significant when analyzing individual countries. Horn et al. (2014) demonstrated that the AMF community composition in a small area harboring high plant diversity and different abiotic soil conditions was not affected by environmental effects, concluding that biotic factors such as AMF-plant interactions were more influential. Because the core-species community of potato was conserved over a wide range of environmental conditions, our study also supports this. On the other hand, Lugo et al. (2008, 2012) reported that increasing altitude had a negative impact on AMF richness and diversity, which is in contrast to reports from the Tibetan Plateau, where Gai et al. (2012) showed that AMF diversity did not change with increasing elevation. Zangaro et al. $(2008,2013)$ showed that root colonization and spore density decreased among successional stages from grasslands to mature forests and different stages in plant age and succession also influence the AMF communities (Husband et al. 2002; Aldrich-Wolfe 2007). Other studies indicate host preferences (Scheublin et al. 2004; Sýkorová et al. 2007; Torrecillas et al. 2012; Yang et al. 2012). In general, standardized analyses, possibly based on the methods presented here, may allow a more robust interpretation and comparison of such data in the future.

\section{Comparison with a clone library Sanger sequencing approach}

From the clone library previously established from the same Peruvian DNA extracts as used here, the most abundant potato root colonizers were an unknown Claroideoglomus sp. (Sp. 5), F. mosseae (Sp. 12) and R. irregularis (Sp. 9). From the 454 relative abundance data for Peru, the most abundant colonizers (from lowest to highest) were Claroideoglomus sp. (Sp. 5), Rhizophagus invermaius (Sp. 35; not previously found in the clone library) and the previously found Acaulospora sp. (Sp. 14). Two species, Ambispora sp. (Sp. 1) and Archaeospora sp. (Sp. 4) annotated from the clone library were not found by 454 sequencing. Because we could annotate other Ambispora and Archaeospora species, we hypothesize that these species perhaps were present but their sequences incorrectly affiliated to very closely related species after the automatic alignment of the 454 sequences. Using different approaches and targeting different fungal communities, Kauserud et al. (2012) and Tedersoo et al. (2010) observed that some of the most abundant OTUs obtained in 454 sequencing were not recovered in the clone library or found in low amounts. In our case it became clear that there are strong limitations when using a clone-based Sanger sequencing approach, but nevertheless, the utility of long sequences, especially for unknown species, should not be overlooked as they provide the phylogenetic context for 454 sequence taxonomic affiliation.

In conclusion, for a better understanding of the dynamics of AMF communities the characterization of their species is necessary. This is especially important to compare among different studies by ecologically meaningful diversity patterns. The improvement of pyrosequencing methods with the goal of obtaining longer reads will eventually permit accurate species annotation. Using $\sim 760$ bp reads combined with the EPA approach allowed us to annotate sequences to both unknown and described species with high resolution. Even though the analyzed samples were from sites with highly variable environmental conditions, we could identify the members of a conserved potato AMF core species community, composed of two Acaulospora spp., C. nodosa and an unknown Claroideoglomus sp., usually accompanied by one or more Rhizophagus species. The identification and characterization of the yet unnamed AMF species associated with food crops will facilitate a selective design of AMF inoculum and application schemes with the purpose of improving sustainable agricultural practices. 
Acknowledgments The research leading to these results has received funding from the European Community's Seventh Framework Programme FP7/2007 under grant agreement no. 227522. We would like to thank people who were involved in the sampling coordinated by Juan Pablo Suárez and Paul Lojan in Ecuador, Javier Franco in Bolivia and Mercy Rojas in Peru.

Conflict of Interest The authors declare no conflict of interest.

Open Access This article is distributed under the terms of the Creative Commons Attribution License which permits any use, distribution, and reproduction in any medium, provided the original author(s) and the source are credited.

\section{References}

Aldrich-Wolfe L (2007) Distinct mycorrhizal communities on new and established hosts in a transitional tropical plant community. Ecology 88:559-566

Alguacil MM, Díaz-Pereira E, Caravaca F, Fernández DA, Roldán A (2009) Increased diversity of arbuscular mycorrhizal fungi in a long-term field experiment via application of organic amendments to a semiarid degraded soil. Appl Environ Microbiol 75:4254-4263

Amend AS, Seifert KA, Bruns TD (2010) Quantifying microbial communities with 454 pyrosequencing: does read abundance count? Mol Ecol 19:5555-5565

Anders S, Huber W (2010) Differential expression analysis for sequence count data. Genome Biol 11:R106

Bakker MG, Tu ZJ, Bradeen JM, Kinkel LL (2012) Implications of pyrosequencing error correction for biological data interpretation. PLoS ONE 7:e44357

Bálint M, Schmidt PA, Sharma R, Thines M, Schmitt I (2014) An Illumina metabarcoding pipeline for fungi. Ecol Evol 4:2642-2653

Berger SA, Stamatakis A (2011) Aligning short reads to reference alignments and trees. Bioinformatics 27:2068-2075

Berger SA, Krompass D, Stamatakis A (2011) Performance, accuracy, and web server for evolutionary placement of short sequence reads under maximum-likelihood. Systematic Biol 60:291-302

Bharadwaj DP, Lundquist P-O, Alström S (2007) Impact of plant species grown as monocultures on sporulation and root colonization by native arbuscular mycorrhizal fungi in potato. Appl Soil Ecol 35: 213-225

Birch PJ, Bryan G, Fenton B, Gilroy E, Hein I, Jones J, Prashar A, Taylor M, Torrance L, Toth I (2012) Crops that feed the world 8: potato: are the trends of increased global production sustainable? Food Sec 4: 477-508

Brundrett MC (2009) Mycorrhizal associations and other means of nutrition of vascular plants: understanding the global diversity of host plants by resolving conflicting information and developing reliable means of diagnosis. Plant Soil 320:37-77

Caporaso JG, Kuczynski J, Stombaugh J, Bittinger K, Bushman FD, Costello EK, Fierer N, Pena AG, Goodrich JK, Gordon JI et al (2010) QIIME allows analysis of high-throughput community sequencing data. Nat Methods 7:335-336

Cesaro P, van Tuinen D, Copetta A, Chatagnier O, Berta G, Gianinazzi S, Lingua G (2008) Preferential colonization of Solanum tuberosum L. roots by the fungus Glomus intraradices in arable soil of a potato farming area. Appl Environ Microbiol 74:5776-5783

Cline MS, Smoot M, Cerami E, Kuchinsky A, Landys N, Workman C, Christmas R, Avila-Campilo I, Creech M, Gross B, Hanspers K et al
(2007) Integration of biological networks and gene expression data using cytoscape. Nat Protoc 2:2366-2382

R Core Team (2014) R: A language and environment for statistical computing. R Foundation for Statistical Computing, Vienna, Austria. URL http://www.R-project.org/

Davies JFT, Calderón CM, Huaman Z, Gómez R (2005) Influence of a flavonoid (formononetin) on mycorrhizal activity and potato crop productivity in the highlands of Peru. Sci Hortic 106:318-329

Davison J, Öpik M, Zobel M, Vasar M, Metsis M, Moora M (2012) Communities of arbuscular mycorrhizal fungi detected in forest soil are spatially heterogeneous but do not vary throughout the growing season. PLoS One 7:e41938

Dechassa N, Schenk MK, Claassen N, Steingrobe B (2003) Phosphorus efficiency of cabbage (Brassica oleraceae L. var. capitata), carrot (Daucus carota L.), and potato (Solanum tuberosum L.). Plant Soil 250:215-224

Dumbrell AJ, Ashton PD, Aziz N, Feng G, Nelson M, Dytham C, Fitter AH, Helgason T (2011) Distinct seasonal assemblages of arbuscular mycorrhizal fungi revealed by massively parallel pyrosequencing. New Phytol 190:794-804

Edgar RC (2010) Search and clustering orders of magnitude faster than BLAST. Bioinformatics 26:2460-2461

FAO (2012) Food and Agriculture Organization of the United Nations, Land Resources. FAOSTATS-Crops. http://faostat.fao.org/site/567/ default.aspx\#ancor Accessed 16 June 2014

Gai JP, Tian H, Yang FY, Christie P, Li XL, Klironomos JN (2012) Arbuscular mycorrhizal fungal diversity along a Tibetan elevation gradient. Pedobiol 55:145-151

Gamper HA, Walker C, Schüßler A (2009) Diversispora celata sp. nov: molecular ecology and phylotaxonomy of an inconspicuous arbuscular mycorrhizal fungus. New Phytol 182:495-506

Hack H, Gall H, Klemke T, Klose R, Meier U, Stauss R, Witzenberger A (1993) Phänologische entwicklungsstadien der kartoffel (Solanum tuberosum L.). codierung und beschreibung nach der erweiterten BBCH-skala mit abbildungen. Nachrichtenbl Deut Pflanzenschutzd 45:11-19

Han M, Zmasek C (2009) phyloXML: XML for evolutionary biology and comparative genomics. BMC Bioinformatics 10:356

Horn S, Caruso T, Verbruggen E, Rillig MC, Hempel S (2014) Arbuscular mycorrhizal fungal communities are phylogenetically clustered at small scales. ISME J doi:10.1038/ismej.2014.72

Husband R, Herre EA, Young JPW (2002) Temporal variation in the arbuscular mycorrhizal communities colonising seedlings in a tropical forest. FEMS Microbiol Ecol 42:131-136

Katoh K, Misawa K, Kuma K, Miyata T (2002) MAFFT: a novel method for rapid multiple sequence alignment based on fast fourier transform. Nucleic Acids Res 30:3059-3066

Kauserud H, Kumar S, Brysting A, Nordén J, Carlsen T (2012) High consistency between replicate 454 pyrosequencing analyses of ectomycorrhizal plant root samples. Mycorrhiza 22:309-315

Kivlin SN, Hawkes CV, Treseder KK (2011) Global diversity and distribution of arbuscular mycorrhizal fungi. Soil Biol Biochem 43:2294 2303

Kohout P, Sudová R, Janoušková M, Čtvrtlíková M, Hejda M, Pánková H, Slavíková R, Štajerová K, Vosátka M, Sýkorová Z (2014) Comparison of commonly used primer sets for evaluating arbuscular mycorrhizal fungal communities: is there a universal solution? Soil Biol Biochem 68:482-493

Kõljalg U, Nilsson RH, Abarenkov K, Tedersoo L, Taylor AFS, Bahram M, Bates ST, Bruns TD, Bengtsson-Palme J, Callaghan TM et al (2013) Towards a unified paradigm for sequence-based identification of fungi. Mol Ecol 22:5271-5277

Krüger M, Stockinger H, Krüger C, Schüßler A (2009) DNA-based species level detection of Glomeromycota: one PCR primer set for all arbuscular mycorrhizal fungi. New Phytol 183:212-223 
Krüger M, Krüger C, Walker C, Stockinger H, Schüßler A (2012) Phylogenetic reference data for systematics and phylotaxonomy of arbuscular mycorrhizal fungi from phylum to species level. New Phytol 193:970-984

Lekberg Y, Schnoor T, Kjøller R, Gibbons SM, Hansen LH, Al-Soud WA, Sørensen SJ, Rosendahl S (2012) 454-sequencing reveals stochastic local reassembly and high disturbance tolerance within arbuscular mycorrhizal fungal communities. J Ecol 100:151-160

Lekberg Y, Gibbons SM, Rosendahl S (2014) Will different OTU delineation methods change interpretation of arbuscular mycorrhizal fungal community patterns? New Phytol 202:1101-1104

Li X, Gai J, Cai X, Li X, Christie P, Zhang F, Zhang J (2014) Molecular diversity of arbuscular mycorrhizal fungi associated with two cooccurring perennial plant species on a Tibetan altitudinal gradient. Mycorrhiza 24:95-107

Lindahl BD, Nilsson RH, Tedersoo L, Abarenkov K, Carlsen T, Kjøller R, Kõljalg U, Pennanen T, Rosendahl S, Stenlid J et al (2013) Fungal community analysis by high-throughput sequencing of amplified markers - a user's guide. New Phytol 199:288-299

Liu Y, He J, Shi G, An L, Öpik M, Feng H (2011) Diverse communities of arbuscular mycorrhizal fungi inhabit sites with very high altitude in Tibet Plateau. FEMS Microbiol Ecol 78:355-365

Lugo MA, Ferrero M, Menoyo E, Estévez MC, Siñeriz F, Anton A (2008) Arbuscular mycorrhizal fungi and rhizospheric bacteria diversity along an altitudinal gradient in South American Puna grassland. Microb Ecol 55:705-713

Lugo MA, Negritto MA, Jofré M, Anton A, Galetto L (2012) Colonization of native Andean grasses by arbuscular mycorrhizal fungi in puna: a matter of altitude, host photosynthetic pathway and host life cycles. FEMS Microbiol Ecol 81:455-466

Maherali H, Klironomos JN (2007) Influence of phylogeny on fungal community assembly and ecosystem functioning. Science 316 : 1746-1748

McArthur DAJ, Knowles NR (1992) Resistance responses of potato to vesicular-arbuscular mycorrhizal fungi under varying abiotic phosphorus levels. Plant Physiol 100:341-351

McMurdie PJ, Holmes S (2014) Waste not, want not: why rarefying microbiome data is inadmissible. PLoS Comput Biol 10:e1003531

Mummey DL, Rillig MC (2007) Evaluation of LSU rRNA-gene PCR primers for analysis of arbuscular mycorrhizal fungal communities via terminal restriction fragment length polymorphism analysis. J Microbiol Methods 70:200-204

Oehl F, Sýkorová Z, Redecker D, Wiemken A, Sieverding E (2006) Acaulospora alpina, a new arbuscular mycorrhizal fungal species characteristic for high mountainous and alpine regions of the Swiss Alps. Mycologia 98:286-294

Oehl F, Silva GA, Palenzuela J, Sánchez-Castro I, Castillo C, Sieverding E (2011) Acaulospora punctata, a new fungal species in the Glomeromycetes from mountainous altitudes of the Swiss Alps and Chilean Andes. Nova Hedwigia 93:353-362

Oksanen J, Guillaume-Blanchet F, Kindt R, Legendre P, Minchin PR, O'Hara RB, Simpson GL, Solymos P, Henry M, Stevens H, Wagner H (2013) vegan: Community ecology package. R package version 2.0-10. http://CRAN.R-project.org/package=vegan

Öpik M, Metsis M, Daniell TJ, Zobel M, Moora M (2009) Large-scale parallel 454 sequencing reveals host ecological group specificity of arbuscular mycorrhizal fungi in a boreonemoral forest. New Phytol 184:424-437

Öpik M, Zobel M, Cantero J, Davison J, Facelli J, Hiiesalu I, Jairus T, Kalwij J, Koorem K, Leal M et al (2013) Global sampling of plant roots expands the described molecular diversity of arbuscular mycorrhizal fungi. Mycorrhiza 23:411-430

Redecker D (2000) Specific PCR primers to identify arbuscular mycorrhizal fungi within colonized roots. Mycorrhiza 10:73-80

Redecker D, Schüßler A, Stockinger H, Stürmer S, Morton J, Walker C (2013) An evidence-based consensus for the classification of arbuscular mycorrhizal fungi (Glomeromycota). Mycorrhiza 23: 515-531

Scheublin TR, Ridgway KP, Young JPW, van der Heijden MGA (2004) Nonlegumes, legumes, and root nodules harbor different arbuscular mycorrhizal fungal communities. Appl Environ Microbiol 70:6240 6246

Schmidt SK, Sobieniak-Wiseman LC, Kageyama SA, Halloy SRP, Schadt CW (2008) Mycorrhizal and dark-septate fungi in plant roots above 4270 meters elevation in the Andes and Rocky Mountains. Arct Antarct Alp Res 40:576-583

Schoch C, Seifert K, Huhndorf S, Robert V, Spouge J, Levesque C, Chen W, Bolchacova E, Voigt K, Crous P, Miller A et al (2012) Nuclear ribosomal internal transcribed spacer (ITS) region as a universal DNA barcode marker for Fungi. Proc Natl Acad Sci U S A 109: 6241-6246

Senés-Guerrero C, Torres-Cortés G, Pfeiffer S, Rojas M, Schüßler A (2014) Potato-associated arbuscular mycorrhizal fungal communities in the Peruvian Andes. Mycorrhiza 24:405-417

Smith SE, Read DJ (2008) Mycorrhizal symbiosis 3rd edn. Academic, London

Spooner DM, McLean K, Ramsay G, Waugh R, Bryan GJ (2005) A single domestication for potato based on multilocus amplified fragment length polymorphism genotyping. Proc Natl Acad Sci U S A 102:14694-14699

Stockinger H, Walker C, Schüßler A (2009) "Glomus intraradices DAOM197198', a model fungus in arbuscular mycorrhiza research, is not Glomus intraradices. New Phytol 183:1176-1187

Stockinger H, Krüger M, Schüßler A (2010) DNA barcoding of arbuscular mycorrhizal fungi. New Phytol 187:461-474

Sun Y, Cai Y, Huse SM, Knight R, Farmerie WG, Wang X, Mai V (2012) A large-scale benchmark study of existing algorithms for taxonomyindependent microbial community analysis. Brief Bioinfom 13: $107-121$

Sýkorová Z, Wiemken A, Redecker D (2007) Co-occurring Gentiana verna and Gentiana acaulis and their neighboring plants in two Swiss upper montane meadows harbor distinct arbuscular mycorrhizal fungal communities. Appl Environ Microbiol 73:5426-5434

Tawaraya K, Hirose R, Wagatsuma T (2012) Inoculation of arbuscular mycorrhizal fungi can substantially reduce phosphate fertilizer application to Allium fistulosum L. and achieve marketable yield under field condition. Biol Fertil Soils 48:839-843

Tedersoo L, Nilsson RH, Abarenkov K, Jairus T, Sadam A, Saar I, Bahram M, Bechem E, Chuyong G, Kõljalg U (2010) 454 Pyrosequencing and Sanger sequencing of tropical mycorrhizal fungi provide similar results but reveal substantial methodological biases. New Phytol 188:291-301

Tilman D, Cassman KG, Matson PA, Naylor R, Polasky S (2002) Agricultural sustainability and intensive production practices. Nature 418:671-677

Torrecillas E, Alguacil MM, Roldán A (2012) Host Preferences of arbuscular mycorrhizal fungi colonizing annual herbaceous plant species in semiarid mediterranean prairies. Appl Environ Microbiol 78:6180-6186

Urcelay C, Acho J, Joffre R (2011) Fungal root symbionts and their relationship with fine root proportion in native plants from the Bolivian Andean highlands above 3,700 m elevation. Mycorrhiza 21:323-330

van der Heijden MGA, Richard DB, van Straalen NM (2008) The unseen majority: soil microbes as drivers of plant diversity and productivity in terrestrial ecosystems. Ecol Lett 11:296-310

van Loon LC (2007) Plant responses to plant growth-promoting rhizobacteria. Eur J Plant Pathol 119:243-254

Yang H, Zang Y, Yuan Y, Tang J, Chen X (2012) Selectivity by host plants affects the distribution of arbuscular mycorrhizal fungi: evidence from ITS rDNA sequence metadata. BMC Evolut Biol 12:50 
Zangaro W, Assis R, Rostirola L, Souza P, Gonçalves M, Andrade G, Nogueira M (2008) Changes in arbuscular mycorrhizal associations and fine root traits in sites under different plant successional phases in southern Brazil. Mycorrhiza 19:3745
Zangaro W, Rostirola L, Souza P, Almeida Alves R, Lescano L, Rondina A, Nogueira M, Carrenho R (2013) Root colonization and spore abundance of arbuscular mycorrhizal fungi in distinct successional stages from an Atlantic rainforest biome in southern Brazil. Mycorrhiza 23:221-233 